\title{
A prediction model for the grade of liver fibrosis using magnetic resonance elastography
}

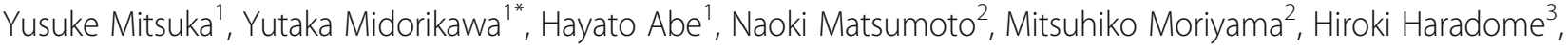 \\ Masahiko Sugitani ${ }^{4}$, Shingo Tsuji ${ }^{5}$ and Tadatoshi Takayama'
}

\begin{abstract}
Background: Liver stiffness measurement (LSM) has recently become available for assessment of liver fibrosis. We aimed to develop a prediction model for liver fibrosis using clinical variables, including LSM.

Methods: We performed a prospective study to compare liver fibrosis grade with fibrosis score. LSM was measured using magnetic resonance elastography in 184 patients that underwent liver resection, and liver fibrosis grade was diagnosed histologically after surgery. Using the prediction model established in the training group, we validated the classification accuracy in the independent test group.

Results: First, we determined a cut-off value for stratifying fibrosis grade using LSM in 122 patients in the training group, and correctly diagnosed fibrosis grades of 62 patients in the test group with a total accuracy of $69.3 \%$. Next, on least absolute shrinkage and selection operator analysis in the training group, LSM $(r=0.687, P<0.001)$, indocyanine green clearance rate at $15 \mathrm{~min}$ (ICGR15) $(r=0.527, P<0.001)$, platelet count $(r=-0.537, P<0.001)$ were selected as variables for the liver fibrosis prediction model. This prediction model applied to the test group correctly diagnosed 32 of 36 (88.8\%) Grade I (F0 and F1) patients, 13 of 18 (72.2\%) Grade II (F2 and F3) patients, and 7 of $8(87.5 \%)$ Grade III (F4) patients in the test group, with a total accuracy of $83.8 \%$.

Conclusions: The prediction model based on LSM, ICGR15, and platelet count can accurately and reproducibly predict liver fibrosis grade.
\end{abstract}

Keywords: Liver fibrosis, Prediction model, Liver stiffness measurement, Magnetic resonance elastography

\section{Background}

It is clinically important to diagnose the grade of fibrosis in patients with chronic liver disease. Accurate assessment of liver fibrosis is necessary to determine the indications for treatment of hepatitis $\mathrm{C}$ virus infection using direct-acting antivirals [1-3] or interferon therapy $[4,5]$. In liver resection, the presence of cirrhosis is associated with blood loss and severe postoperative complications, especially in major liver resection [6]. Therefore, assessment of the extent of fibrosis provides a means to predict surgical risks in patients undergoing liver resection [7].

\footnotetext{
*Correspondence: mido-tky@umin.ac.jp

'Department of Digestive Surgery, Nihon University Faculty of Medicine, 30-1

Oyaguchi Kami-machi, Tokyo, Itabashi-ku 173-8610, Japan

Full list of author information is available at the end of the article
}

Percutaneous core-needle biopsy remains the gold standard and has been widely used to evaluate the cause or grade of liver fibrosis in patients with several liver diseases or abnormalities [8]. Although histological diagnosis of biopsy specimens can provide direct diagnostic information, percutaneous liver biopsy is contraindicated in such patients with a tendency to easy bleeding, ascites, or difficult approach for biopsy by ultrasonography.

Recently, liver stiffness measurement (LSM) has become a standard method for assessing liver fibrosis in patients with chronic liver disease [9-12]. Instead of core-needle biopsy, LSM using transient elastography by ultrasound (TE) [9] or magnetic resonance elastography (MRE) [10-12] is a novel, noninvasive, and reproducible method for assessing liver fibrosis to allow treatment. It was reported that LSM using MRE was significantly 
correlated with the pathological grade of advanced fibrosis in patients with nonalcoholic fatty liver disease [10-12]. Furthermore, LSM by TE was available to predict the risk for liver resection for hepatocellular carcinoma by stratifying the fibrosis of the background liver [13].

This study was performed to establish a prediction model to enable estimation of liver fibrosis based on selected clinical variables, and thereafter confirm the accuracy of our algorithm.

\section{Methods}

\section{Inclusion and exclusion criteria}

Patients that underwent liver resection for malignant tumors in Nihon University Itabashi Hospital from 2014 to 2016 were included in this study. Patients were divided into training and test groups (2:1 ratio). Each participant provided written, informed consent, and this study was approved by the institutional review board of Nihon University (protocol number: RK-141209-4). All clinical investigations were conducted according to the principles of the Declaration of Helsinki.

Patients were included if they were $\geq 20$ years of age, candidates for liver resection due to cancer, and provided written informed consent. Patients fulfilling the exclusion criteria were excluded from the study as described previously [14].

\section{Liver stiffness measurement}

MRE was performed as described previously [14]. Briefly, low-frequency $(60 \mathrm{~Hz})$ mechanical shear waves with amplitude of $70 \%$ were applied to the liver with a proprietary passive driver placed over the right upper quadrant of the abdominal wall for MRE acquisition. After MRE scanning, axial wave and elastogram map images were generated to evaluate quantitative liver stiffness in kilopascals $[15,16]$.

One radiologist with 10 years of experience, blinded to the histopathology results and all clinical data, measured the liver stiffness by placing the region of interest in the right lobe of the liver on the elastogram maps using the average from four hepatic slice locations.

\section{Pathology}

The degree of fibrosis of resected specimens was determined in accordance with the New Inuyama Classification by two pathologists with more than 5 years of experience in the field of liver pathology [17]. The degree of fibrosis was scored as F0 (no fibrosis), F1 (fibrous portal expansion), F2 (bridging fibrosis), F3 (bridging fibrosis with architectural distortion), or F4 (liver cirrhosis). After pathological diagnosis, the degree of fibrosis was categorized into three grades as follows: no or slight fibrosis, Grade I (containing F0 and F1); moderate fibrosis, Grade II (containing F2 and F3); and liver cirrhosis, Grade III (containing F4).

The degree of necroinflammatory activity was scored as A0 (no necroinflammatory reaction), A1 (mild), A2 (moderate), or A3 (severe). Steatosis was graded according to the Brunt scoring system as follows: 0, none; 1 , steatosis in $1 \%-33 \%$ of hepatocytes; 2 , steatosis in $33 \%-66 \%$ of hepatocytes; 3 , steatosis in $66 \%-100 \%$ of hepatocytes [18].

\section{Statistical analysis}

The correlation coefficient for liver fibrosis was calculated using the Spearman's rank test. Independent factors for the prediction of liver fibrosis were identified using least absolute shrinkage and selection operator (LASSO) analysis, and the prediction model was established by multiple logistic regression model based on these variables. The predictive ability of fibrosis score was assessed by receiver operating characteristic (ROC) curve analysis and the corresponding area under the curve (AUC).

All statistical analyses were performed using the JMP 12.0.1 statistical software package (SAS Institute Inc., Cary, NC). In all analyses, $P<0.05$ was taken to indicate statistical significance.

\section{Results}

\section{Patients}

The 122 patients enrolled in the first two thirds of the study period were selected for the training group, and the remaining 62 patients in the second one third were selected as the test group (Fig. 1). Eighty-one patients (44.0\%) had viral hepatitis. The liver function of 178 patients (96.7\%) was Child-Pugh classification A, and the median indocyanine green clearance rate at $15 \mathrm{~min}$ (ICGR15) was $12.2 \%$ (range: $3.4 \%-33.0 \%$ ) (Table 1 ). The indications for liver resection are summarized in Table 2.

\section{Pathology of the liver}

After the operation, 44 (23.9\%), 59 (32.0\%), 26 (14.1\%), $26(14.1 \%)$, and $29(15.7 \%)$ patients were pathologically diagnosed with liver fibrosis degree F0, F1, F2, F3, and F4, respectively, and 103 (55.9\%), 52 (28.2\%), and 29 (15.7\%) patients were classified into Grade I, Grade II, and Grade III, respectively. Other histological findings including necroinflammatory activity grades and steatosis grades are summarized in Table 3.

\section{Prediction of liver fibrosis by LSM value}

First, we determined a cut-off value for classifying the fibrosis grade using only the LSM value in 122 patients in the training group. The medians of LSM were 0.65 (range, -0.17 to 2.14 ), 1.25 (0.42 to 3.33 ), and 2.58 (0.64 to 3.90) in Grade I, Grade II, and Grade III, 


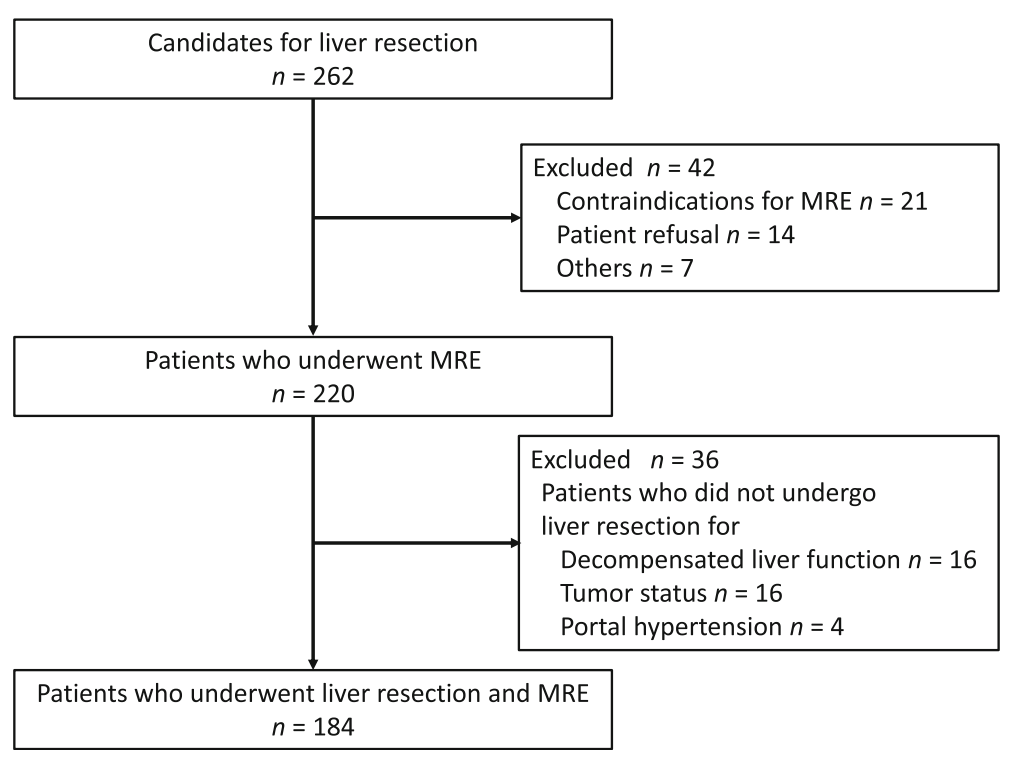

Fig. 1 Flow diagram describing patient recruitment and follow-up

respectively, in the training group. The cut-off value was determined as the average of the median LSM score of each Grade: Grade I, $\leq 0.95$; Grade II, 0.951.91; Grade III, $\geq 1.91$. Using the cut-off value, 26 of 36 (72.2\%) Grade I patients, 10 of 18 (55.5\%) Grade II patients, and 7 of 8 (87.5\%) Grade III patients were correctly diagnosed among the 62 patients in the independent test group, with an accuracy of $69.3 \%$ in total.

\section{Prediction of liver fibrosis by fibrosis score}

In the training group, liver fibrosis was significantly correlated with LSM value $(\mathrm{r}=0.687, \quad P<0.001)$, ICGR15 ( $\mathrm{r}=0.527, P<0.001)$, platelet count $(\mathrm{r}=-0.537$, $P<0.001)$, hyaluronic acid $(\mathrm{r}=0.433, P<0.001)$, and IV core $7 \mathrm{~S}(\mathrm{r}=0.464, P<0.001)$ on Spearman's rank test (Fig. 2). On LASSO analysis, LSM, ICGR15, and platelet count were selected as variants for the liver fibrosis prediction model.

Using the three variants, the formula that predicts the fibrosis grade of the liver was established as follows:

Fibrosis $\quad$ Score $=0.428 \quad \mathrm{X}_{\text {Elast }}+0.038 \quad \mathrm{X}_{\mathrm{ICGR} 15}-0.042$ $\mathrm{X}_{\text {Plt }}-0.025$.

$\mathrm{X}_{\text {Elast, }}$ LSM value by MRE (kilopascal).

$\mathrm{X}_{\text {ICGR15 }}$, ICGR15 value (\%).

$\mathrm{X}_{\mathrm{Plt}}$, serum platelet count $\left(10^{9} / \mathrm{L}\right)$.

Based on this prediction model, the median fibrosis scores in patients with Grade I, Grade II, and Grade III in the training group were 0.66 (range, -0.49 to 2.08), 1.24 (0.13 to 3.38), and 2.74 (1.23 to 4.87), respectively $(P<0.001)$. Then, we defined the cut-off value of each Grade as the average of each median fibrosis score in the training group: Grade 1, $\leq 0.95$; Grade 2, 0.95-1.99; and Grade 3, $\geq 1.99$ (Fig. 3a).
Next, we applied this prediction model to the 62 independent patients in the test group, and the median fibrosis scores in patients with Grade I, Grade II, and Grade III were 0.51 (range, -0.45 to 1.84 ), 1.44 ( 0.39 to 3.33 ), and 2.81 (0.92 to 3.15), respectively. According to the definition, 32 of 36 (88.8\%) Grade I patients, 13 of 18 (72.2\%) Grade II patients, and 7 of 8 (87.5\%) Grade III patients were correctly diagnosed in the independent test group, with an accuracy of $83.8 \%$ in total (Fig. 3b). The ROC curve for fibrosis grade in relation to the fibrosis score was shown in Additional file 1: Figure S1, and the AUC of the ROC was 0.930 (Fibrosis grade I vs II/III) and 0.925 (Fibrosis grade I/II vs III).

\section{Discussion}

This study showed that the grade of liver fibrosis was noninvasively, accurately, and reproducibly determined using three clinical variables, i.e., LSM, ICGR15, and platelet count. Our prediction model is available to decide the management for patients with chronic liver disease.

There have been several previous reports predicting advanced liver fibrosis using prediction scores combining demographic and clinical routine tests [19-21]. However, as the contribution of LSM by MRE to estimate liver fibrosis was high on multiple logistic regression analyses in this study, a prediction score consisting only of conventional serum markers is considered not to be sufficient for correct diagnosis of liver fibrosis.

Consistent with previous reports, LSM value using MRE was significantly correlated with liver fibrosis in this study. In the distribution of liver fibrosis, the threshold of LSM value was determined using receiver operating characteristic curve analysis between the two groups 
Table 1 Patient characteristics

\begin{tabular}{|c|c|}
\hline Characteristic & Patients $(n=184)$ \\
\hline Age, yr & $69(42-91)$ \\
\hline Male, n (\%) & $130(70.6)$ \\
\hline \multicolumn{2}{|l|}{ Background liver disease, $n(\%)$} \\
\hline Non-B non-C liver & $103(55.9)$ \\
\hline Hepatitis B & $35(19.0)$ \\
\hline Hepatitis C & $46(25.0)$ \\
\hline Body mass index, $\mathrm{kg} / \mathrm{m}^{2}$ & $22.8(14.4-37.1)$ \\
\hline \multicolumn{2}{|l|}{ ASA grading, $n(\%)$} \\
\hline Grade 1 & $65(35.3)$ \\
\hline Grade 2 & $116(63.0)$ \\
\hline Grade 3 & $3(1.6)$ \\
\hline History of hepatic resection, n (\%) & $48(26.0)$ \\
\hline Hemoglobin, g/dL & $13.0(8.2-16.9)$ \\
\hline White blood cell count, $10^{9} / \mathrm{L}$ & $5.1(2.3-12.1)$ \\
\hline Platelet count, $10^{9} / \mathrm{L}$ & $17.2(4.8-43.5)$ \\
\hline Total bilirubin, mg/dL & $0.59(0.24-1.48)$ \\
\hline Aspartate aminotransferase, U/L & $28(11-170)$ \\
\hline Alanine aminotransferase, $\mathrm{U} / \mathrm{L}$ & $23(6-271)$ \\
\hline PT-INR & $0.99(0.85-1.36)$ \\
\hline Albumin, $g / L$ & $3.9(2.3-4.8)$ \\
\hline Hyaluronic acid, ng/mL & $62(12-737)$ \\
\hline IV core $7 \mathrm{~S}, \mathrm{ng} / \mathrm{mL}$ & $5.8(3.2-13.0)$ \\
\hline \multicolumn{2}{|l|}{ Child-Pugh classification, n (\%) } \\
\hline A & $178(96.7)$ \\
\hline B & $6(3.2)$ \\
\hline ICGR15, \% & $12.2(3.4-33.0)$ \\
\hline LSM value by MRE, $\mathrm{kPa}$ & $3.3(1.4-11.2)$ \\
\hline
\end{tabular}

Data were presented as median (range), if not specified ASA indicates American Society of Anesthesiology, PT-INR Prothrombin time-International normalized ratio, ICGR15 Indocyanine green clearance rate at $15 \mathrm{~min}$, LSM Liver stiffness measurement, MRE Magnetic resonance elastography

of liver fibrosis. As a result, the cut-off value of LSM for classifying fibrosis stages $0-2$ and 3-4 in nonalcoholic fatty liver disease could be clearly determined $[11,12]$. In these reports, MRE-based LSM could predict advanced liver fibrosis, but not cirrhosis, using only MRE-based LSM.

Table 2 Diseases for liver resection

\begin{tabular}{ll}
\hline Disease, $n(\%)$ & Patients $(n=184)$ \\
\hline Hepatocellular carcinoma & $107(58.1)$ \\
Intrahepatic cholangiocarcinoma & $11(5.9)$ \\
Hilar bile duct cancer & $4(2.1)$ \\
Gallbladder cancer & $4(2.1)$ \\
Cystoadenocarcinoma $_{\text {Metastatic liver cancer }}^{\mathrm{a}}$ & $1(0.5)$ \\
\hline
\end{tabular}

${ }^{a}$ From 57 colorectal cancer, two gastric cancer, two pancreatic cancer, and one neuroendocrine tumor
Table 3 Liver pathology

\begin{tabular}{ll}
\hline & Patients $(n=184)$ \\
\hline Steatosis, $n$ (\%) & $129(70.1)$ \\
$0(0 \%)$ & $44(23.9)$ \\
1 (1 to 33\%) & $10(5.4)$ \\
2 (33 to 66\%) & $1(0.5)$ \\
3 (66 to 100\%) & \\
Lobular inflammation, $n$ (\%) & $41(22.2)$ \\
A0 (no necro-inflammatory reaction) & $99(53.8)$ \\
A1 (mild) & $43(23.3)$ \\
A2 (moderate) & $1(0.5)$ \\
A3 (severe) & \\
Fibrosis, n (\%) & $44(23.9)$ \\
F0 (no fibrosis) & $59(32.0)$ \\
F1 (fibrous portal expansion) & $26(14.1)$ \\
F2 (bridging fibrosis) & $26(14.1)$ \\
F3 (bridging fibrosis with architectural distortion) \\
F4 (liver cirrhosis) & $29(15.7)$ \\
\hline
\end{tabular}

In addition to LSM value, we selected factors contributing to liver fibrosis using LASSO analysis and improved the prediction model containing LSM, ICGR15, and platelet count. Based on these variables, we established a formula to classify chronic liver disease into three grades, and validated the results using an independent patient group. As a result, the accuracy rate of the diagnosis of liver fibrosis grade was remarkably improved compared with the cut-off value by LSM only. Especially, our prediction model has advantages for diagnosis of liver cirrhosis and normal liver.

Our prediction model could predict the normal liver or low grade of fibrosis (F0 and F1) and liver cirrhosis (F4) with high accuracy in the test patient group. On the other hand, the accuracy rate of diagnosis of moderate fibrosis (F2 and F3) by the prediction model was not so high compared with other fibrosis grades. This is because the range of LSM value of F3 stage was wide, which made the prediction of moderate fibrosis difficult. Similarly, LSM by MRE could not clearly divide F1 and F2 in previous reports $[11,12,22]$, suggesting that LSM is vulnerable to distinguish the moderate grade of liver fibrosis.

In addition to MRE, LSM using TE is considered the standard method for assessing liver fibrosis [9, 15, 23]. However, only one-directional measurement can be performed in TE including reflection and refraction. In addition, the area measured in the liver using TE is relatively small, which causes sampling variability due to heterogeneity of advanced fibrosis. In contrast, the twodimensional displacement vector was assessed in MRE [23]. In fact, LSM by MRE was more accurate than that of 

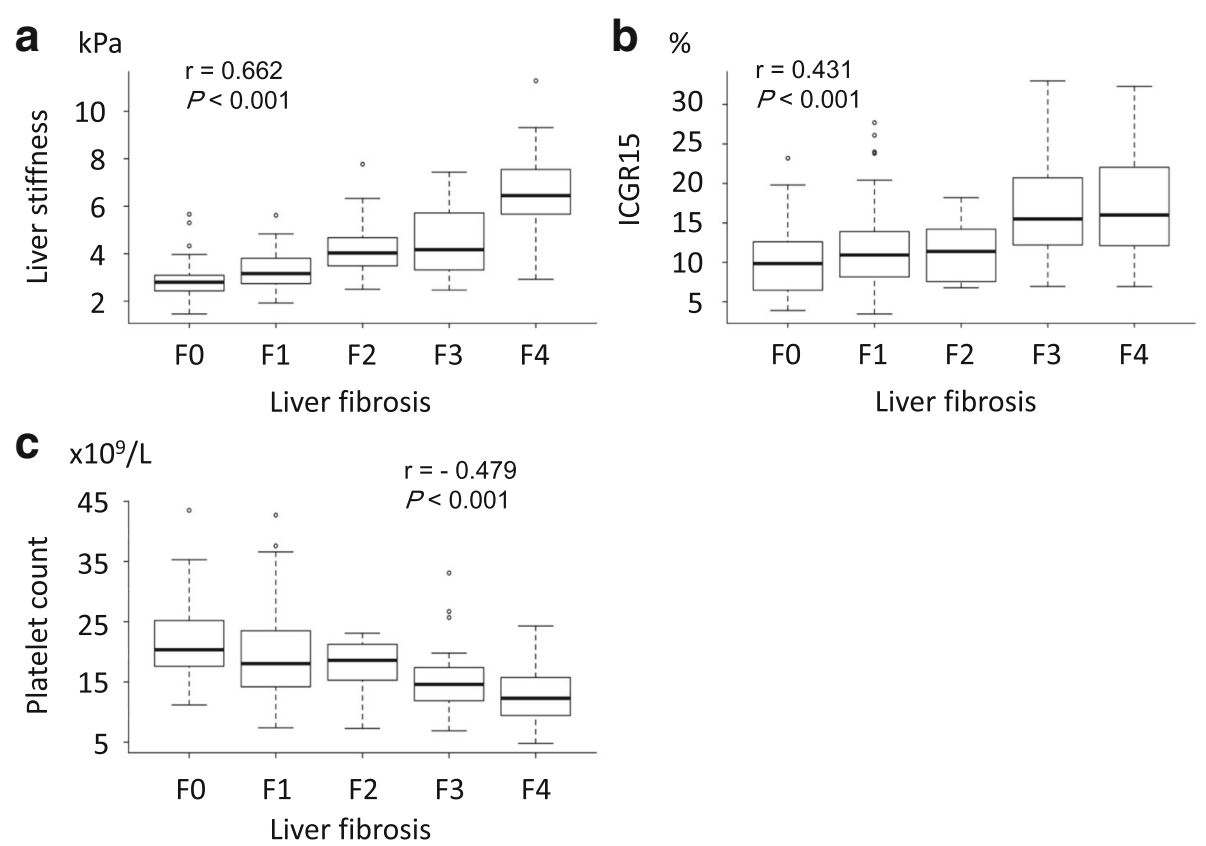

Fig. 2 Distribution of LSM by MRE, ICGR15, and platelet count, and liver fibrosis as shown by box plots. There were significant correlations between LSM (a), ICGR15 (b), and platelet count (c) and the pathological grade of liver fibrosis

TE in identification of liver fibrosis [12, 24, 25]. Thus, MRE is more objective, has greater reproducibility, and has better diagnostic accuracy than TE for liver fibrosis.

We usually decide the type of surgical procedure for hepatocellular carcinoma according to liver function, including ICGR15 and serum total bilirubin level [26]. In addition to the diagnosis of liver fibrosis without percutaneous liver biopsy, LSM using TE or MRE could predict the risk of postoperative complications due to blood loss [13]. Furthermore, LSM using MRE could serve as a postoperative predictor of liver regeneration in patients with liver cirrhosis undergoing right hepatectomy [27]. As the surgical outcomes are dependent on massive bleeding during surgery [28] and liver regeneration after resection [29], LSM could provide more accurate decision criteria for selection of the surgical procedure for patients with chronic liver disease.

\section{Conclusions}

We established a model for prediction of liver fibrosis based on ICGR15 and platelet count in addition to LSM by MRE. In contrast to previous reports, our model consists of multiple variables, which could diagnose liver cirrhosis without percutaneous biopsy. It would be available for determination of not only medical treatments but also indications for liver resection.
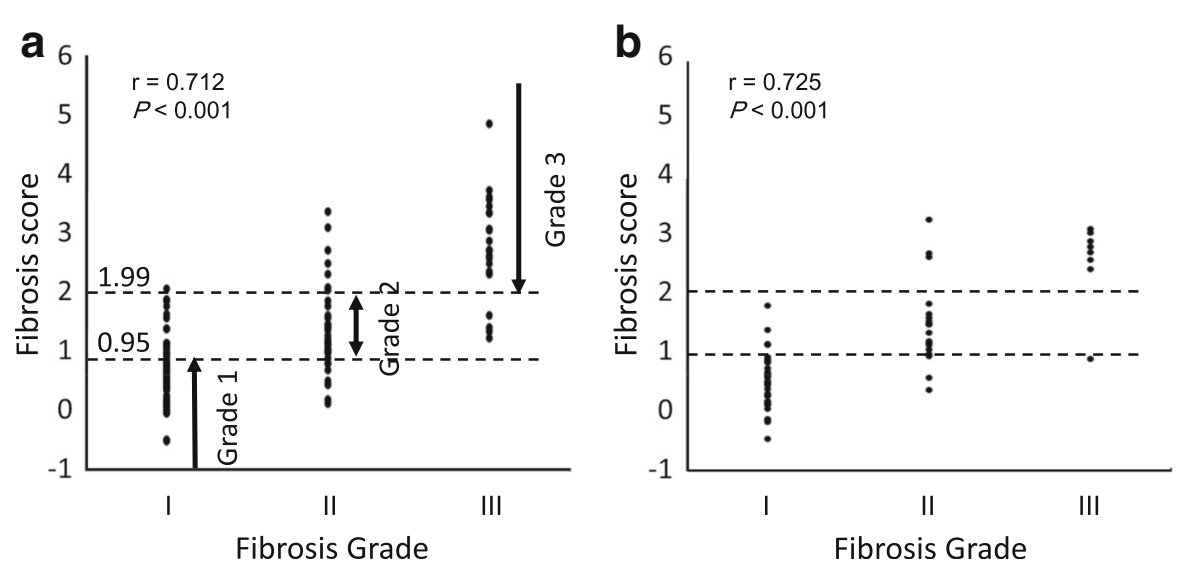

Fig. 3 Distributions of fibrosis score and fibrosis grade. a Distributions of fibrosis score and fibrosis grade in the 122 patients in the training group $(r=0.712, P<0.001)$. b Distributions of fibrosis score and fibrosis grade in the 62 patients in the test group $(r=0.725, P<0.001)$ 


\section{Additional file}

Additional file 1: Figure S1. ROC analysis for fibrosis score in relation to fibrosis grade. (a) Fibrosis grade I vs II/III. The AUC of the ROC was 0.930 (b) Fibrosis grade I/II vs III. The AUC of the ROC was 0.925. (PPTX $64 \mathrm{~kb}$ )

\section{Abbreviations}

AUC: Area under the curve; ICGR15: Indocyanine green clearance rate at 15 min; LASSO: Least absolute shrinkage and selection operator; LSM: Liver stiffness measurement; MRE: Magnetic resonance elastography; ROC: Receiver operating characteristic; TE: Transient elastography by ultrasound

\section{Acknowledgments}

Not applicable

\section{Funding}

This work was mainly supported by a Grant-in-Aid for Scientific Research (C) 15 K10152 from the Ministry of Education, Culture, Sports, Science and Technology (MEXT), Japan.

\section{Availability of data and materials}

Data and materials analyzed in thie study are not available due to the pretection of individual privacy, but are available from the corresponding author on reasonable request.

\section{Authors' contributions}

All authors have read and approved the final version of this manuscript. Y.M.: Designed the study, data acquisition, and wrote the initial draft of the manuscript; Y.M.: Conceived of the study, and participated in its design and coordination and helped to draft the manuscript; H.A., N.M.: Acquired the data; S.T.: Performed the statistical analysis; H.H.: Revised the article for the magnetic resonance elastography content; M.M., M.S., T.T.: Revised the article for clinical content

\section{Ethics approval and consent to participate}

Each participant provided written, informed consent, and this study was approved by the institutional review board of Nihon University (protocol number: RK-141209-4)

\section{Consent for publication}

Not applicable

\section{Competing interests}

The authors delcare that they have no competing interests.

\section{Publisher's Note}

Springer Nature remains neutral with regard to jurisdictional claims in published maps and institutional affiliations.

\section{Author details \\ 'Department of Digestive Surgery, Nihon University Faculty of Medicine, 30-1 Oyaguchi Kami-machi, Tokyo, Itabashi-ku 173-8610, Japan. ${ }^{2}$ Department of Gastroenterology and Hepatology, Nihon University Faculty of Medicine, 30-1 Oyaguchi Kami-machi, Tokyo, Itabashi-ku 173-8610, Japan. ${ }^{3}$ Department of Radiology, Nihon University Faculty of Medicine, 30-1 Oyaguchi Kami-machi, Tokyo, Itabashi-ku 173-8610, Japan. ${ }^{4}$ Department of Pathology, Nihon University Faculty of Medicine, 30-1 Oyaguchi Kami-machi, Tokyo, Itabashi-ku 173-8610, Japan. ${ }^{5}$ Research Center of Advanced Science and Technology, Genome Science Division, University of Tokyo, 4-6-1 Komaba, Tokyo, Meguro-ku 153-8904, Japan.}

Received: 15 July 2017 Accepted: 20 November 2017 Published online: 28 November 2017

\section{References}

1. Bourliere M, Benali S, Ansaldi C, Le Folgoc G, Riso A, Lecomte L. Optimal therapy of genotype-2 chronic hepatitis C: what's new? Liver Int. 2015 35(Suppl 1):21-6.
2. Horsmans Y, Berg T, Desager JP, Mueller T, Schott E, Fletcher SP, et al. Isatoribine, an agonist of TLR7, reduces plasma virus concentration in chronic hepatitis C infection. Hepatology. 2005:42:724-31.

3. Martin NK, Vickerman P, Dore GJ, Grebely J, Miners A, Cairns J, et al. Prioritization of HCV treatment in the direct-acting antiviral era: an economic evaluation. J Hepatol. 2016;65:17-25.

4. Chung RT, Gale M Jr, Polyak SJ, Lemon SM, Liang TJ, Hoofnagle JH. Mechanisms of action of interferon and ribavirin in chronic hepatitis C: summary of a workshop. Hepatology. 2008;47:306-20.

5. Levin S, Hahn T. Interferon system in acute viral hepatitis. Lancet. 1982;1:592-4

6. Poon RT, Fan ST, Lo CM, Liu CL, Lam CM, Yuen WK, et al. Extended hepatic resection for hepatocellular carcinoma in patients with cirrhosis: is it justified? Ann Surg. 2002;236:602-11.

7. Kim MS, Lee JR. Assessment of liver stiffness measurement: novel intraoperative blood loss predictor? World J Surg. 2013:37:185-91.

8. Bravo AA, Sheth SG, Chopra S. Liver biopsy. N Engl J Med. 2001;344:495-500.

9. Castera L, Forns X, Alberti A. Non-invasive evaluation of liver fibrosis using transient elastography. J Hepatol. 2008;48:835-47.

10. Huwart L, Sempoux C, Vicaut E, Salameh N, Annet L, Danse E, et al. Magnetic resonance elastography for the noninvasive staging of liver fibrosis. Gastroenterology. 2008;135:32-40.

11. Loomba R, Wolfson T, Ang B, Hooker J, Behling C, Peterson M, et al. Magnetic resonance elastography predicts advanced fibrosis in patients with nonalcoholic fatty liver disease: a prospective study. Hepatology. 2014; 60:1920-8.

12. Yin M, Glaser KJ, Talwalkar JA, Chen J, Manduca A, Ehman RL, Hepatic MR. Elastography: clinical performance in a series of 1377 consecutive examinations. Radiology. 2016;278:114-24.

13. Wong JS, Wong GL, Chan AW, Wong WW, Cheung YS, Chong CN, et al. Liver stiffness measurement by transient elastography as a predictor on posthepatectomy outcomes. Ann Surg. 2013;257:922-8.

14. Abe H, Midorikawa $Y$, Mitsuka $Y$, Aramaki O, Higaki T, Matsumoto N, et al. Predicting postoperative outcomes of liver resection by magnetic resonance elastography. Surgery. 2017;162:248-55.

15. Kim D, Kim WR, Talwalkar JA, Kim HJ, Ehman RL. Advanced fibrosis in nonalcoholic fatty liver disease: noninvasive assessment with MR elastography. Radiology. 2013;268:411-9.

16. Venkatesh SK, Yin M, Ehman RL. Magnetic resonance elastography of liver: technique, analysis, and clinical applications. J Magn Reson Imaging. 2013; 37:544-55.

17. Ichida F, Tsuji T, Omata M, Ichida T, Inoue K, Kamimura T, et al. New Inuyama classification; new criteria for histological assessment of chronic hepatitis. Int Hepatol Commun. 1996;6:112-9.

18. Brunt EM, Janney CG, Di Bisceglie AM, Neuschwander-Tetri BA, Bacon BR. Nonalcoholic steatohepatitis: a proposal for grading and staging the histological lesions. Am J Gastroenterol. 1999;94:2467-74.

19. Ratziu V, Giral P, Charlotte F, Bruckert E, Thibault V, Theodorou I, et al. Liver fibrosis in overweight patients. Gastroenterology. 2000;118:1117-23.

20. Rosenberg WM, Voelker M, Thiel R, Becka M, Burt A, Schuppan D, et al. Serum markers detect the presence of liver fibrosis: a cohort study. Gastroenterology. 2004;127:1704-13.

21. Sterling RK, Lissen E, Clumeck N, Sola R, Correa MC, Montaner J, et al. Development of a simple noninvasive index to predict significant fibrosis in patients with HIV/HCV coinfection. Hepatology. 2006;43:1317-25.

22. Yoshimitsu K, Mitsufuji T, Shinagawa Y, Fujimitsu R, Morita A, Urakawa H, et al. MR elastography of the liver at 3.0 T in diagnosing liver fibrosis grades; preliminary clinical experience. Eur Radiol. 2016;26:656-63.

23. Ichikawa S, Motosugi U, Morisaka H, Sano K, Ichikawa T, Tatsumi A, et al. Comparison of the diagnostic accuracies of magnetic resonance elastography and transient elastography for hepatic fibrosis. Magn Reson Imaging. 2015;33:26-30.

24. Park CC, Nguyen P, Hernandez C, Bettencourt R, Ramirez K, Fortney L, et al. Magnetic resonance Elastography vs transient Elastography in detection of fibrosis and noninvasive measurement of Steatosis in patients with biopsyproven nonalcoholic fatty liver disease. Gastroenterology. 2017;152:598-607 e2.

25. Batheja M, Vargas $H$, Silva AM, Walker F, Chang $Y H$, De Petris G, et al. Magnetic resonance elastography (MRE) in assessing hepatic fibrosis: performance in a cohort of patients with histological data. Abdom Imaging. 2015;40:760-5.

26. Makuuchi M, Kosuge T, Takayama T, Yamazaki S, Kakazu T, Miyagawa S, et al. Surgery for small liver cancers. Semin Surg Oncol. 1993;9:298-304. 
27. Jang $\mathrm{S}$, Lee JM, Lee $\mathrm{DH}$, Joo I, Yoon JH, Chang W, et al. Value of MR elastography for the preoperative estimation of liver regeneration capacity in patients with hepatocellular carcinoma. J Magn Reson Imaging. 2017;45: $1627-36$.

28. Aramaki O, Takayama T, Higaki T, Nakayama H, Ohkubo T, Midorikawa Y, et al. Decreased blood loss reduces postoperative complications in resection for hepatocellular carcinoma. J Hepatobiliary Pancreat Sci. 2014;21:585-91.

29. Nagasue N, Yukaya H, Ogawa Y, Kohno H, Nakamura T. Human liver regeneration after major hepatic resection. A study of normal liver and livers with chronic hepatitis and cirrhosis. Ann Surg. 1987;206:30-9.

Submit your next manuscript to BioMed Central and we will help you at every step:

- We accept pre-submission inquiries

- Our selector tool helps you to find the most relevant journal

- We provide round the clock customer support

- Convenient online submission

- Thorough peer review

- Inclusion in PubMed and all major indexing services

- Maximum visibility for your research

Submit your manuscript at www.biomedcentral.com/submit
Biomed Central 